\title{
A convenient category of topological partial groups
}

\author{
A. Fathy
}

Correspondence:

afa_math@yahoo.com

Department of Mathematics,

Faculty of Science, Al-Azhar

University, Nasr City 11884, Cairo,

Egypt

\begin{abstract}
In this paper, the concept of $\wp$-continuous map is introduced and some of their basic properties are discussed. Also, the category $\mathbf{K}$, of topological partial groups, as objects and the $\wp$-morphisms of topological partial groups, as arrows, is introduced, which is alternative to the category $\mathbf{K}$, of topological spaces, as objects and k-continuous maps, as arrows, and satisfies the same nice properties of the category $\mathbf{k p g}$, of $k$-partial groups, as objects, and the morphisms of $\underline{k}$-partial groups, as arrows (Abd- Allah et al., J. Egyption Math. Soc 25:276-278, 2017).
\end{abstract}

Keywords: Partial group, Partial group homomorphism, Topological group, Topological partial group, $k$-partial group

MSC: 22A05, 22A10, 22A20, 54H11

\section{Introduction}

In [1], A.M. Abd- Allah et al. introduced the concept of topological partial groups and discussed some of their basic properties. Also, they introduced the category Tpg of topological partial groups, as objects and the homorphisms of topological partial groups, as arrows. So, the category $\mathbf{T p g}$ has the following deficiencies:

(i) If $a \in S$, then the right transformation $r_{a}: S \rightarrow S, x \mapsto x a$ and the left transformation $l_{a}: S \rightarrow S, x \mapsto a x$, may not be open.

(ii) The quotient map $\rho_{N}: S \rightarrow S, x \mapsto x N, N \leq S$, may not be open, in general, where $S / N$ has the identification topology with respect to the quotient map.

(iii) $\quad$ Let $S$ be a topological partial group and $N \unlhd S$. Then, the partial group $S / N$ may not be a topological partial group, since the cartesian product of two identification maps may not be identification.

In [2], A.M. Abd- Allah et al. introduced the concept of $k$-partial groups and discussed some of their basic properties. Also, they introduced the category $\mathbf{k p g}$, of $\underline{k}$-partial groups, as objects, and the morphisms of $\underline{k}$-partial groups, as arrows which is modified the above deficiencies. In this paper, the concept of $\wp$-continuous maps is introduced and some of their basic properties are discussed. Also, the category $\mathbf{K}$ of topological partial groups, as objects, and the $\wp$-morphisms of topological partial groups , as arrows, is introduced, which is alternative to the category $\mathbf{K}$, of topological spaces, as objects, and $k$-continuous maps, as arrows. The category $\mathbf{K}$ satisfied the same nice properties of the category $\mathbf{k p g}$. The idea of $\wp$-continuous maps was taken from the definition of $k$-continuous map [3].

(c) The Author(s). 2017 Open Access This article is distributed under the terms of the Creative Commons Attribution 4.0 International License (http://creativecommons.org/licenses/by/4.0/), which permits unrestricted use, distribution, and reproduction in any medium, provided you give appropriate credit to the original author(s) and the source, provide a link to the Creative Commons license, and indicate if changes were made. 


\section{Preliminaries}

We collect for sake of reference the needed definitions and results appeared in the given references.

Definition 1 [4] Let $S$ be a semigroup. Then, $x \in S$ is called an idempotent element if $x \cdot x=x$. The set of all idempotent elements in $S$ is denoted by $E(S)$.

Definition 2 [5] Let $S$ be a semigroup and $x \in S$. Then, an element $e \in S$ is called a partial identity of $x$ if:

(i) $e x=x e=x$,

(ii) If $e^{\prime} x=x e^{\prime}=x$, for some $e^{\prime} \in S$, then $e e^{\prime}=e^{\prime} e=e$.

Theorem 1 [5] Let S be a semigroup. Then,

(i) If $x \in S$ has a partial identity, then it is unique

(ii) $E(S)$ is the set of all partial identities of the elements of $S$.

We will denote by $e_{x}$ the partial identity of the element $x \in S$.

Definition 3 [5] Let $S$ be a semigroup and $x \in S$ has a partial identity element $e_{x}$. Then, $y \in S$ is called a partial inverse of $x$ if:

(i) $x y=y x=e_{x}$,

(ii) $e_{x} y=y e_{x}=y$.

We will denote the partial inverse $y$ of $x \in S$ by $x^{-1}$.

Definition 4 [5] A semigroup $S$ is called a partial group if:

(i) Every $x \in S$ has a partial identity $e_{x}$

(ii) Every $x \in S$ has a partial inverse $x^{-1}$

(iii) The map $e_{S}: S \rightarrow S, x \mapsto e_{x}$ is a semigroup homomorphism

(iv) The map $\gamma: S \rightarrow S, x \mapsto x^{-1}$ is a semigroup antihomomorphism.

So, every group is a partial group.

Definition 5 [6] Let S be a partial group and $x \in S$. Then, we define $S_{x}=\left\{y \in S: e_{x}=e_{y}\right\}$.

Theorem 2 [5] Let $S$ be a partial group and $x \in S$. Then,

(i) $S_{x}$ is a maximal subgroup of $S$ which has identity $e_{x}$

(ii) $S=\bigcup\left\{S_{x}: x \in S\right\}$.

Corollary 1 [5] Every partial group is a disjoint union of a family of groups.

Definition 6 [3] Let $X$ be a topological space. Then, the map $\alpha: C \rightarrow X$ is called a test map if $\alpha$ is continuous and $C$ is a compact Hausdorff space.

Definition 7 [3] Let $X$ and $Y$ be topological spaces. Then, the map $f: X \rightarrow Y$ is called $k$-continuous iff $\alpha: C \rightarrow Y$ is continuous, for each test map $\alpha: C \rightarrow X$. 
Let $\tau$ be the category of topological spaces, as objects and continuous maps, as arrows. Also, let $\mathbf{K}$ be the category of topological spaces, as objects and $k$-continuous maps, as arrows. It is clear that the category $\boldsymbol{\tau}$ is a wide subcategory of $\mathbf{K}$.

Definition 8 [1] Let $S$ be a partial group and $\tau$ be a topology on S. Then, $S$ is called a topological partial group if the following maps are continuous:

(i) The product map: $\mu: S \times S \rightarrow S,(x, y) \mapsto x y$

(ii) The partial identity map: $e_{S}: S \rightarrow S, x \mapsto e_{x}$

(iii) The partial inverse map: $\gamma: S \rightarrow S, x \mapsto x^{-1}$.

Definition 9 [1] Let $S$ be a topological partial group and $a \in S$. Then, the map $r_{a}: S \rightarrow$ $S, x \mapsto x a$ is called a right transformation and the map $l_{a}: S \rightarrow S, x \mapsto$ ax is called a left transformation.

Theorem 3 [1] The maps $r_{a}$ and $l_{a}$ are continuous.

Let $\wp$ be a non-empty full subcategory of $\tau$ which satisfies the following conditions [7]:

(i) If $A$ is a closed subspace of an object $B$ of $\wp$, then $A$ is a $k \wp$-space.

(ii) If $B$ and $C$ are objects in $\wp$, then $B \times C$ is also object in $\wp$.

(iii) For objects $X$ in $\wp$ and $Y$ in $\boldsymbol{\tau}$, the evaluation map $e: Y^{X} \times X \rightarrow Y,(f, x) \mapsto f(x)$ and $x \in X$, is continuous, where $Y^{X}$ has the compact open topology.

(iv) If $A$ and $B$ are objects in $\wp$, then the topological sum $A \sqcup B$ is also an object in $\wp$.

Definition 10 [2] Let $S$ be a topological partial group. Then, the map $h: C \rightarrow S$ is called a $\wp$-test map if $h$ is continuous and $h^{-1}\left(S_{e_{x}}\right)$ is open in $C$ for each $e_{x} \in E(S)$, where $C \in \operatorname{obj}(\wp)$.

\section{$\wp$-continuous maps}

In this section, the notion of $\wp$-continuous map is introduced and some of their basic properties are discussed. Also, the category $\mathbf{K}$ of $\wp$-continuous maps, as objects and the morphisms of $\wp$-continuous maps as arrows, is introduced.

Definition 11 Let $S$ and $T$ be topological partial groups. Then, the map $f: S \rightarrow T$ is called $\wp$-continuous iffh $: C \rightarrow T$ is continuous, for each a $\wp$-test map $h: C \rightarrow S$.

We note that every continuous map of topological partial group is $\wp$-continuous. So, the following maps are $\wp$-continuous:

(i) The identity map $I: S \rightarrow S$

(ii) The partial identity map: $e_{S}: S \rightarrow S, x \mapsto e_{x}$

(iii) The partial inverse map: $\gamma: S \rightarrow S, x \mapsto x^{-1}$

(iv) The maps $r_{a}$ and $l_{a}$.

Definition 12 Let $f: S \rightarrow T$ be a $\wp$-continuous map. Then, $f$ is called a $\wp-$ morphism if it is a partial group homomorphism.

We note that (i) and (iii) above are $\wp$-morphisms. 
Theorem 4 Iff $: S \rightarrow T$ and $g: T \rightarrow F$ are $\wp$-morphisms, then $g f: S \rightarrow F$ is also $a$ §-morphism.

Proof It is clear that $g f$ is a partial group homomorphism. Let $h: C \rightarrow S$ be a $\wp$-test map. Since $f$ is $\wp$-continuous, then $f h: C \rightarrow T$ is continuous. Now, $(f h)^{-1}\left(T_{e}\right)=$ $h^{-1}\left(f^{-1}\left(T_{e}\right)\right)$, for each $e \in E(T)$. Since $f$ is a partial group homomorphism, then $f^{-1}\left(T_{e}\right)$ is a maximal subgroup of $S$. So, $(f h)^{-1}\left(T_{e}\right)$ is open in $C$, for each $e \in E(T)$. That means that $f h$ is a $\wp$-test map. Since $g$ is $\wp$-continuous, then $g(f h)=(g f) h: C \rightarrow F$ is continuous. Then, $g f$ is $\wp$-continuous. Hence, $g f$ is a $\wp$-morphism.

Definition 13 A subset $V$ of the topological partial group $S$ is called $\wp$-open if $h^{-1}[V]$ is open in $C$ for each a $\wp$-test map $h: C \rightarrow S$

From the above definition, we have that $S_{e_{x}}$ is $\wp$-open in $S$.

Theorem 5 The family $\left\{\wp-\tau_{S}\right\}$ of $\wp$-open sets form a topology on $S$.

Proof It is clear that $\phi$ and $S$ are $\wp$-open sets, since $h^{-1}[S]=C$ and $h^{-1}[\phi]=\phi$. If $U$ and $V$ are $\wp$-open sets, then $h^{-1}[U]$ and $h^{-1}[V]$ are open sets in C. But $h^{-1}[U \cap V]=$ $h^{-1}[U] \cap h^{-1}[V]$ is open in C. So, $U \cap V$ is a $\wp$-open set. Similarly, let $\left(U_{\lambda}\right)_{\lambda \in L}$ be a subfamily of $\wp$-open sets. Then, $h^{-1}\left[U_{\lambda}\right]$ are open in C, for each $\lambda \in L$. Since $h^{-1}\left[\bigcup_{\lambda} U_{\lambda}\right]=$ $\bigcup_{\lambda} h^{-1}\left[U_{\lambda}\right]$ is open in C. Hence, $\bigcup U_{\lambda}$ is a $\wp$-open set.

Definition 14 A subset $A$ of the topological partial group $S$ is called a §-neighbourhood of $x \in S$ if there exists a ø-open set $U$ in $S$ such that $x \in U \subseteq A$.

The family of all $\wp$-neighbourhoods of $x \in S$ is called a $\wp$-neighbourhood system and is denoted by $\wp-N_{x}$

Proposition $1 A$ subset $A \subseteq S$ of the topological partial group $S$ is a $\wp$-open set if and only if it is a $\wp$-neighbourhood of each of its points.

Proof Let $A$ be a $\wp$-open set. Then, $x \in A \subseteq A$, for all $x \in A$. Hence, $A$ is a $\wp$ neighbourhood of $x$. Conversely, for each $x \in A$, there exists a $\wp$-open set $U_{x}$ such that $x \in U_{x} \subseteq A$. So, $A=\bigcup_{x \in A} U_{x}$. Hence, $A$ is a $\wp$-open set.

Theorem 6 Let $S$ be a topological partial group and $x \in S$. Then,

(i) $x \in N$, for all $N \in N_{x}$

(ii) If $N \in N_{x}$ and $N \subseteq M$, then $M \in N_{x}$

(iii) If $N, M \in N_{x}$, then $N \cap M \in N_{x}$

(iv) If $N \in N_{x}$, then there exists $M \in N_{x}$ such that $N \in N_{y}$, for each $y \in M$.

Proof (i) If $N \in N_{x}$, then there exists a $\wp$-open set $U$ in $S$ such that $x \in U \subseteq N$.

Hence, $x \in N$.

(ii) If $N \in N_{x}$, then there exists a $\wp$-open set $U$ in $S$ such that $x \in U \subseteq N$. Since, $N \subseteq M$, then $x \in U \subseteq M$. Hence, $M \in N_{x}$.

(iii) If $N, M \in N_{x}$, then there exist two $\wp$-open sets $U$ and $V$, respectively such that $x \in U \subseteq N$ and $x \in V \subseteq M$. So, we have that $x \in U \cap V \subseteq N \bigcap M$. Since $N \cap M$ is a $\wp$-open set, then $N \bigcap M \in N_{x}$. 
(iv) If $N \in N_{x}$, then there exists a $\wp$-open set $M$ in $S$ such that $x \in M \subseteq N$. Since $M$ is a $\wp$-open set, then $M \in N_{y}$, for all $y \in M$. Since, $N \subseteq M$, then $N \in N_{y}$, for each $y \in M$.

Definition 15 Let $S$ be a topological partial group and $A \subseteq S$. Then, $x \in A$ is called $a$ $\wp$-interior point of $A$ if $A$ is a $\wp$-neighbourhood of $x$.

The set of all $\wp$-interior points of $A$ is called $\wp$-interior set and is denoted by $\wp-A^{0}$.

Proposition 2 Let $S$ be a topological partial group and $A, B \subseteq S$. Then,

(i) $\wp-A^{0} \subseteq A$

(ii) If $A \subseteq B$, then $\wp-A^{0} \subseteq \wp-B^{0}$

(iii) $\wp-A^{0}$ is a $\wp$-open set

(iv) $\quad\left(\wp-A^{0}\right)^{0}=\wp-A^{0}$.

Proof (i) Let $x \in \wp-A^{0}$. Then, $A \in N_{x}$. So, $x \in A$.

(ii) Let $x \in \wp-A^{0}$. Then, $A \in N_{x}$. Since, $A \subseteq B$, then $B \in N_{x}$ and so $x \in \wp-B^{0}$. Hence, $\wp-A^{0} \subseteq \wp-B^{0}$.

(iii) Let $x \in \wp-A^{0}$. Then, $A \in N_{x}$. Thus, there exists $N \in N_{x}$ such that $A \in N_{y}$, for all $y \in N$. That is, $y \in \wp-A^{0}$, for all $y \in N$. Hence, $N \subseteq A$. Thus, $x \in N \subseteq \wp-A^{0}$. So, $A \in N_{x}$. Therefore, $\wp-A^{0}$ is a $\wp$-open set.

(iv) Since $\wp-A^{0} \subseteq A$, then from (ii) $\left(\wp-A^{0}\right)^{0} \subseteq \wp-A^{0}$. It remains that $\wp-A^{0} \subseteq\left(\wp-A^{0}\right)^{0}$. This is given from $x \in \wp-A^{0}$. That is, $\wp-A^{0} \in N_{x}$. Hence, $x \in\left(\wp-A^{0}\right)^{0}$.

Corollary $2 A$ subset $A$ of the topological partial group $S$ is $९$-open if and only if $\wp-$ $A^{0}=A$.

Proof It is obvious.

Definition 16 A subset $A$ of the topological partial group $S$ is called $\wp$-closed if $S-A$ is a ९-open set.

Definition 17 Let $S$ be a topological partial group and $A \subseteq S$. Then, $x \in S \in$ is called a $\wp$-closure point of $A$ if $A \bigcap N \neq \phi$, for each $N \in \wp-N_{x}$.

The set of all $\wp$-closure points of $A$ is called the $\wp$-closure of $A$ and is written by $\wp-\bar{A}$.

Proposition 3 Let $A$ be a subset of the topological partial group $S$. Then, the family $\tau_{A}=\{U \cap A: U$ is $\wp-$ open in $S\}$ is a topology on $A$, which is called $\wp$-relative topology.

Proof It is clear that $\phi, A \in \tau_{A}$ since $\phi=\phi \bigcap A$ and $A=A \bigcap S$. Let $M, N \in \tau_{A}$. Then, there exist two $\wp$-open sets $U$ and $V$ such that $M=U \bigcap A$ and $N=V \bigcap A$. So, $M \bigcap N \in$ $\tau_{A}$. Also, let $V=\left(V_{\lambda}\right)_{\lambda \in L}$ be a subfamily of $\tau_{A}$. Then, for each $\lambda$, there are $\wp$-open sets $U_{\lambda}$ such that $V=U_{\lambda} \bigcap A$. Then, $V=\bigcup_{\lambda \in L} V_{\lambda}=\bigcup_{\lambda \in L}\left(U_{\lambda} \bigcap A\right)=\left(\bigcup_{\lambda \in L} U_{\lambda}\right) \bigcap A$.

Theorem 7 Let $f: S \rightarrow T$ be $\wp$-continuous. Then, $f \mid A: A \rightarrow T$ is $\wp$-continuous. 
Proof Let $U \subseteq T$ be $\wp$-open. Now, $(f \mid A)^{-1}(U)=f^{-1}(U) \bigcap A$. Since $f^{-1}(U)$ is a $\wp$-open set in $S$, then $f^{-1}(U)$ is a $\wp$-open in $A$.

Definition 18 Let $S$ be a topological partial group and A be a subpartial group of S. Then, $A$ with the $\wp$-relative topology is a topological partial group, called a topological subpartial group, denoted by $A \leq S$.

Definition 19 Let $S$ and $T$ be topological partial groups and let $(x, y) \in S \times T$. The set $\wp-(S \times T)$, where $M \in N_{x}$ in $S$ and $N \in N_{y}$ in $T$ is called a $\wp$-basic neighbourhood of $(x, y)$.

Definition 20 A subset $U$ of $M \times N$ is called a $\wp$-neighbourhood if there exists a $\wp$-basic neighbourhood $M \times N$ of $(x, y)$ such that $(x, y) \in M \times N \subseteq U$.

We note that if $M$ and $N$ are $\wp$-open sets in the topological partial groups $S$ and $T$, respectively, then $M \times N$ is a $\wp$-basic neighbourhood of any $(x, y) \in M \times N$.

Theorem 8 (i) If $A$ and $B$ are $\wp$-open sets in $S$ and $T$, respectively, then $A \times B$ is also $\wp$-open in $S \times T$

(ii) If $C$ and $D$ are $\wp$-closed sets in $S$ and $T$, respectively, then $C \times D$ is also $\wp$-closed in $S \times T$.

Proof (i) Let $(x, y) \in U \times V$. Then, $x \in U$ and $y \in V$. So, $U \in \wp-N_{x}$ in $S$ and $V \in \wp-N_{y}$ in $T$. This implies $U \times V$ is a $\wp$-basic neighbourhood of $(x, y)$. Since $(x, y) \in U \times V \subseteq A \times B$, then $U \times V \in N_{(x, y)}$. Hence, $A \times B$ is also $\wp$-open in $S \times T$.

(ii) We have $(S \times T)-(C \times D)=(S-C) \times T \bigcup S \times(T-D)$. Since $S-C$ and $T-D$ are $\wp$-open sets in $S$ and $T$, respectively, then $(S-C) \times T$ and $S \times(T-D)$ are $\wp$-open sets in $S \times T$ and so $(S \times T)-(C \times D)$ is $\wp$-open set in $S \times T$. That is, $C \times D$ is $\wp$-closed in $S \times T$.

We note that the following maps are $\wp$-continuous, for each topological partial group $S$ :

(i) The projection maps $P_{1}: S \times T \rightarrow S$ and $P_{2}: S \times T \rightarrow T$.

(ii) The product map $\mu: S \times S \rightarrow S$.

(iii) The diagonal map $\Delta_{S}=\{(x, x): x \in S\}$.

Theorem 9 Iff $: S \rightarrow T$ and $f: S \rightarrow F$ are $\wp$-morphisms, then $(f, g): S \rightarrow T \times F$ is also a §-morphism.

Proof It is clear that $(f, g)$ is a partial group homomorphism. Let $h: C \rightarrow S$ be a $\wp$ test map. Since $f$ is $\wp$-continuous, then $f h: C \rightarrow T$ is continuous. Also, since $g$ is $\wp-$ continuous, then $g h: C \rightarrow T$ is continuous. So, $(f h, g h)=(f, g) h: S \rightarrow T \times F$ is continuous. That is, $(f, g)$ is $\wp$-continuous. Hence, $(f, g)$ is a $\wp$-morphism.

Theorem 10 If $f_{1}: S_{1} \rightarrow T_{1}$ and $f_{2}: S_{2} \rightarrow T_{2}$ are $\wp$-morphisms, then $f_{1} \times f_{2}: S_{1} \times S_{2} \rightarrow$ $T_{1} \times T_{2}$ is also a $\wp$-morphism.

Proof It is clear that $f_{1} \times f_{2}: S_{1} \times S_{2} \rightarrow T_{1} \times T_{2}$ is a partial group homomorphism. Since $f_{1} \times f_{2}=\left(f_{1} P_{1}, f_{2} P_{2}\right)$, then from the last theorem, we have that $f_{1} \times f_{2}$ is $\wp$-continuous. Hence, $f_{1} \times f_{2}$ is a $\wp$-morphism. 
Theorem 11 Let $S$ and $T$ be topological partial groups. Then, the following conditions are equivalent for any map $f: S \rightarrow T$.

(i) $f$ is $\wp$-continuous

(ii) $f^{-1}[U]$ is a $\wp$-open set in $S$ for each $\wp$-open set $U$ in $T$.

(iii) $f^{-1}[U]$ is a $\wp$-closed set in $S$ for each $\wp$-closed set $U$ in $T$.

Proof (i) $\rightarrow$ (ii) Let $f$ be $\wp$-continuous and let $U \subseteq T$ be $\wp$-open. So, $h^{-1}\left[f^{-1}[U]\right]=$ $(f h)^{-1}[U]$ is open in $C$, for each $\wp$-test map $h: C \rightarrow T$. Hence, $f^{-1}[U]$ is a $\wp$-open set in $S$.

(ii) $\rightarrow$ (iii) Let $U$ be $\wp$-closed in $T$. So $T-U$ is $\wp$-open in $T$. Therefore, $f^{-1}[T-U]=$ $S-f^{-1}[U]$ is $\wp$-open in $S$. Hence, $f^{-1}[U]$ is $\wp$-closed in $S$.

(iii) $\rightarrow$ (ii) Let $U$ be $\wp$-open in $T$. So, $T-U$ is $\wp$-closed in $T$. Therefore, $f^{-1}[T-U]=$ $S-f^{-1}[U]$ is $\wp$-closed in $S$. Hence, $f^{-1}[U]$ is $\wp$-open in $S$.

(iii) $\rightarrow$ (i) Let $h: C \rightarrow S$ be a $\wp$-test map and $U \subseteq T$ be open. So, $f^{-1}[U]$ is $\wp$-open in $S$. Therefore, $h^{-1}\left[f^{-1}[U]\right]=(f h)^{-1}[U]$ is open in $C$. Hence, $f$ is $\wp$-continuous.

Definition 21 Let $S$ and $T$ be topological partial groups. Then, the map $f: S \rightarrow T$ is called $\wp$-open if $f(U)$ is $\wp$-open in $T$ for each $\wp$-open set $U$ in $S$. Also, the map $f: S \rightarrow T$ is called $\wp$-closed iff $f(U)$ is $\wp$-closed in $T$ for each $\wp$-closed set $U$ in $S$.

Theorem 12 If $f_{1}: S_{1} \rightarrow T_{1}$ and $f_{2}: S_{2} \rightarrow T_{2}$ are $\wp$-open maps, then $f_{1} \times f_{2}: S_{1} \times S_{2} \rightarrow$ $T_{1} \times T_{2}$ is also a ø-open map.

Proof Let $U \subseteq S_{1} \times T_{1}$ be $\wp$-open and $(x, y) \in U$. Then, there exists a $\wp$-basic neighbourhood $M \times N$ of $(x, y)$ such that $(x, y) \in \wp-(M \times N) \subseteq U$. So, $\left(f_{1} \times f_{2}\right)[M \times N] \subseteq$ $\left(f_{1} \times f_{2}\right)[U]$. Therefore, $f_{1}[M] \times f_{2}[N] \subseteq\left(f_{1} \times f_{2}\right)[U]$. Since $f_{1}$ and $f_{2}$ are $\wp$-open maps, then $f_{1}[M]$ and $f_{2}[N]$ are $\wp$-open sets in $T_{1}$ and $T_{2}$, respectively. Hence, $f_{1} \times f_{2}$ is $\wp$-open.

Theorem 13 The maps $r_{a}$ and $l_{a}$ are §-open maps.

Proof We only prove that $r_{a}$ is $\wp$-open as follows: Let $U \subseteq S$ be $\wp$-open. Then, $U \cap S_{e_{x}}$ is open in the maximal topological subgroup $S_{e_{x}}$ and so is open in $S$. Now, we have two cases:

(i) Let $\left.r_{a}\right|_{S_{e_{x}}}: S_{e_{x}} \rightarrow S_{e_{y}}$. So, $\left.r_{a}\right|_{S_{e_{x}}}\left(U \bigcap S_{e_{x}}\right)=U a \bigcap S_{e_{y}}$. We show that $U a \bigcap S_{e_{y}}$ is open in $S$ as follows: Let $h: C \rightarrow S$ be a $\wp$-test map. Then, $r_{a} h: C \rightarrow S$ is a $\wp$-test map. Now, $\left(r_{a} h\right)^{-1}\left(U a \bigcap S_{e_{y}}\right)=h^{-1}\left(\left(r_{a}\right)^{-1}\left(U a \bigcap S_{e_{y}}\right)\right)=$ $h^{-1}\left(\left(r_{a}\right)^{-1}(U a) \bigcap\left(r_{a}\right)^{-1}\left(S_{e_{y}}\right)\right)=h^{-1}\left(U \cap S_{e_{x}}\right)$. Since $U \cap S_{e_{x}}$ is open in $S$, then $h^{-1}\left(U \cap S_{e_{x}}\right)$ is open in $C$. Hence, $U a \bigcap S_{e_{y}}$ is $\wp$-open in $S$.

(ii) Let $\left.r_{a}\right|_{S_{e_{x}}}: S_{e_{x}} \rightarrow S_{e_{x}}$. Since, the right transformation $\left.r_{a}\right|_{S_{e_{x}}}$ is a homeomorphism of the topological maximal subgroups $S_{e_{x}}$, then $\left.r_{a}\right|_{S_{e_{x}}}\left(U \bigcap S_{e_{x}}\right)$ is open in $S_{e_{x}}$. Since $S_{e_{x}}$ is open in $S$, then $\left.r_{a}\right|_{S_{e_{x}}}\left(U \cap S_{e_{x}}\right)=U a \bigcap S_{e_{x}}$ is open in $S$. That means $r_{a}(U)=\left.\bigcup_{e_{x} \in E(S)} r_{a}\right|_{S_{e_{x}}}\left(U \cap S_{e_{x}}\right)$ is $\wp$-open in $S$.

Similarly, we can prove that $\ell_{a}$ is $\wp$-open.

Theorem 14 Let $S$ be a topological partial group and $A, B \subseteq S$. Then, if $A$ is $\wp-o p e n$ in $S$, then $A B$ and $B A$ are also $\wp$-open in $S$. 
Proof We only prove that $A B$ is $\wp$-open in $S$ as follows: Since $A B=\bigcup_{b \in B} r_{b}(A)$, and $r_{b}(A)$ is $\wp$-open in $S$, then $A B$ is $\wp$-open in $S$. Similarly, we can prove that $B A$ is also $\wp$-open in $S$.

Theorem 15 If $S$ is a topological partial group, then every §-open topological subpartial group of $S$ is $\wp$-closed.

Proof Let $A$ be a $\wp$-open topological subpartial group of $S$. Then, $x A$ is $\wp$-open in $S$, for all $x \in S$. Since $S-A=\bigcup_{x \neq A} x A$, then $S-A$ is $\wp$-open. Therefore, $A$ is $\wp$-closed.

Theorem 16 The projection maps $P_{1}: S \times T \rightarrow S$ and $P_{2}: S \times T \rightarrow T$ are $\wp-o p e n$ maps.

Proof we only prove that $P_{1}$ is $\wp$-open, as follows: let $W \subseteq S \times T$ be $\wp$-open and $x \in$ $P_{1}[W]$. Then, there exists $y \in T$ such that $(x, y) \in W$. Since $W$ is $\wp$-open, then there exists a $\wp$-basic neighbourhood $M \times N$ of $(x, y)$ such that $(x, y) \in M \times N \subseteq W$. So, $x \in M=P_{1}^{-1}[M \times N] \subseteq P_{1}[W]$. Hence, $P_{1}[W] \in \wp-N_{x}$. Therefore, $P_{1}$ is $\wp$-open. Similarly, we can prove that $P_{2}$ is $\wp$-open.

Let $\left\{S_{i}: i=1,2, \cdots, n\right\}$ be a family of topological partial groups and $S=$ $\bigotimes_{i=1}^{n} S_{i}$ be the cartesian product of topological partial groups. That is, $S=$ $\left\{x=\left\langle x_{i}\right\rangle: x_{i} \in S_{i}, \forall i=1,2, \ldots, n\right\}$.

Theorem 17 The partial group $S$ with the cartesian product topology $S=\stackrel{\bigotimes}{i=1}_{i} S_{i}$ is a topological partial group.

Proof The maps $\mu, \gamma$ and $e_{S}$ are $\wp$-continuous, since $\mu=\left\langle\mu_{i}\left(P_{i} \times P_{i}\right)\right\rangle, \gamma=\left\langle\gamma_{i} P_{i}\right\rangle$ and $e_{S}=\left\langle e_{S_{i}} P_{i}\right\rangle$, respectively, where $P_{i}: \bigotimes_{i=1}^{n}\left(S_{i}\right) \rightarrow S_{i}$, are the projection maps.

Definition 22 Let $S$ and $T$ be topological partial groups. A topology $\wp-\tau^{*}$ on $T$ is called $\wp-$ final with respect to the map $f: S \rightarrow T$ if, for any topological partial group $F$ and all maps $g: T \rightarrow F$, we have that $g$ is $\wp$-continuous if $g f: S \rightarrow F$ is $\wp$-continuous.

Theorem 18 The $\wp-\tau^{*}$ final topology on $T$ with respect to the function $f: S \rightarrow T$ exists and is characterized by the following condition: If $U \subseteq T$, then $U$ is $\wp$-open (ø-closed) in

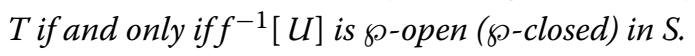

Proof It is clear that $\phi$ and $T$ are $\wp$-open sets in $S$. If $U$ and $V$ are $\wp$-open sets in $T$, then $f^{-1}[U \cap V]=f^{-1}[U] \cap f^{-1}[V]$ is $\wp$-open in $S$. So, $U \cap V$ is $\wp$-open in $T$. Similarly, let $\left(U_{\lambda}\right)_{\lambda \in L}$ be a subfamily of $\wp$-open sets in $T$. Then, $f^{-1}\left[U\left(U_{\lambda}\right)\right]$ are $\wp$-open sets in $S$. So, $\cup U_{\lambda}$ is a $\wp$-open set in $S$. A similar proof applies with $\wp$-open replaced by $\wp$-closed.

Definition 23 Let $S$ and $T$ be topological partial groups. Then, the map $f: S \rightarrow T$ is called $\wp$-identification iff is surjective and Thas the $\wp$-final topology with respect to $f$.

Theorem 19 Let $f: S \rightarrow T$ be a §-continuous surjection. If $f$ is a $\wp$-open (closed) map. Then, $f$ is a $\wp$-identification map.

Proof Let $U \subseteq T$ be a $\wp$-open set. Then, $f^{-1}[U]$ is $\wp$-open in $S$. Since $f$ is surjective, then $f\left[f^{-1}[U]\right]=U$. Hence, $f^{-1}[U]$ is $\wp$-open in $S$ if and only if $U$ is $\wp$-open. A similar proof applies with open replaced by $\wp$-closed. 


\section{Quotients in topological partial groups}

Definition 24 If $S$ is a topological partial group and $N \leq S$, then $S / N$ with the $\wp-$ identification topology, with respect to the quotient map $\rho_{N}: S \rightarrow S / N$, is called the $\wp$-coset space.

Theorem 20 Let $S$ be a topological partial group and $N \leq S$. Then, the quotient map $\rho_{N}: S \rightarrow S / N$ is $\wp$-open.

Proof Let $U \subseteq S$ be open. Then,

$$
\begin{aligned}
\rho_{N}^{-1}\left(\rho_{N}(U)\right) & =\left\{x \in S: \rho_{N}(x) \in \rho_{N}(U)\right\} \\
& =\{x \in S: x N \in U / N\} \\
& =\{x \in S: x \in a N \text { for some } a \in U\} \\
& =\bigcup_{a \in U} a N \\
& =U N .
\end{aligned}
$$

Since $U$ is open in $S$, then $U N$ is open in $S$. Since $\rho_{N}$ is an identification map and $U N$ is open in $S$, then $\rho_{N}(U)$ is open is $S / N$.

Theorem 21 If $S$ is a topological partial group and $N \unlhd S$, then $S / N$ is a topological partial group.

Proof Since $\rho_{N}$ is a $\wp$-open identification map, then $\rho_{N} \times \rho_{N}$ is a $\wp$ identification map. So, the product map $\mu: S / N \times S / N \rightarrow S / N$ is continuous, since $\mu\left(\rho_{N} \times{ }_{k} \rho_{N}\right)=\rho_{N} \mu^{\prime}$, where $\mu^{\prime}: S \times_{k} S \rightarrow S$ is the product map. The partial inverse map $\gamma: S / N \rightarrow S / N$ and the partial identity map $e_{S / N}: S / N \rightarrow S / N$ are continuous, since $\gamma \rho_{N}=\rho_{N} \gamma^{\prime}$ and $e_{S / N} \rho_{N}=\rho_{N} e_{S}$ are $\wp$-continuous and $\rho_{N}$ is an identification map, where $\gamma^{\prime}: S \rightarrow S$, $x \mapsto x^{-1}$ and $e_{S}: S \rightarrow S, x \mapsto e_{x}$ are $\wp$-continuous.

Theorem 22 Let $\varphi: S \rightarrow T$ be an idempotent separating surjective $\wp$-morphism and $K=k e r \varphi$. Then, there exists a unique bijective $\wp$-morphism $\alpha: S / K \rightarrow T$ such that $\varphi=\alpha \rho_{K}$.

Proof It is clear that $\alpha$ is bijective and a partial group homomorphism. Also, $\alpha$ is $\wp$ continuous since $\varphi$ is $\wp$-continuous and $\rho_{K}$ is a $\wp$-identification map.

Theorem 23 Let $S$ be a topological partial group and $M, N \unlhd S$ such that $M \subseteq N$, then

(i) $\quad N / M \unlhd S / M$

(ii) There exists a unique bijective $\wp$-morphism $\alpha:(S / M) /(N / M)$ such that $\rho_{N}=\alpha \rho_{N / M} \rho_{M}$

Proof (i) See [4]

(ii) Let $\rho_{N}: S \rightarrow S / N$ and $\rho_{N}: S \rightarrow S / M$ be the quotient maps. Then, $\rho_{N}$ is an idempotent separating surjective $\wp$-morphism and $\operatorname{ker} \rho_{N}=N$. So, from the last theorem, there exists a unique bijective $\wp$-morphism $\varphi: S / M \rightarrow S / N$ such that $\varphi \rho_{M}=\rho_{N}$. Since $\operatorname{ker} \varphi=N / M$ is a topological partial group, then by the last theorem, there exists a unique bijective $\wp$-morphism $\alpha:(S / M) /(N / M)$, such that $\rho_{N}=\alpha \rho_{N / M} \rho_{M}$. 


\section{Separation axioms.}

Definition 25 Let $S$ be a topological partial group. Then, $S$ is called $\wp$-Hausdorff if, for all $x, y \in S$, there exist $\wp$-open sets $U$ and $V$ such that $x \in U, y \in V$, and $U \cap V \neq \phi$.

Theorem 24 Let $S$ be a topological partial group. Then, $S$ is Hausdorff if and only if $S$ is a $T_{0}$-space.

Proof Let $S$ be a Hausdorff partial group. Then, $S$ is a $T_{0}$-space. Conversely, let $S$ be a $T_{0}$-space. Let $x, y \in S, x \neq y$ :

(i) If $x, y \in S_{a}$, then $S_{a}$ is a $T_{2}$-group and there exist two open sets $U, V$ in $S_{a}$ and also $\wp$-open in $S$ such that $U \cap V \neq \phi$ and $x \in U, y \in V$ and

(ii) If $x \in S_{a}$ and $y \in S_{b}$, then, we have that $S_{a}$ and $S_{b}$ are $\wp$-open and $S_{a} \bigcap S_{b} \neq \phi$. So, $S$ is a Hausdorff partial group.

Theorem 25 Let $S$ be a Hausdorff topological partial group. If $f, g: S \rightarrow T$ are $\wp-$ morphisms of topological partial group, then the difference kernel $A=\{x \in S: f(x)=$ $g(x)\}$ is a $\wp$-closed subpartial group.

Proof $A$ is closed (see [3]). Let $x, y \in A$. Now,

$$
\begin{aligned}
f\left(x y^{-1}\right) & =f(x) f\left(y^{-1}\right) \\
& =f(x) f(y)^{-1} \\
& =g(x) g(y)^{-1}=g\left(x y^{-1}\right) .
\end{aligned}
$$

Therefore, $x y^{-1} \in A$. Hence, $A$ is a $\wp$-closed subpartial group.

Let K'be the category of topological partial groups, as objects and the $\wp$-morphisms, as arrows.

The category $\mathbf{K}$ is a convenient category since this category has a product and a quotient.

Abbreviations

$\mathbf{K}$ : The category of topological partial groups, as objects and the $\wp$-morphisms of topological partial groups, as arrows; $\mathbf{K}$ The category of topological spaces, as objects and k-continuous maps, as arrows; $\mathbf{k p g}$ : The category of $k$-partial groups, as objects, and the morphisms of $k$-partial groups, as arrows; Tpg: The category of topological partial groups, as objects and the homorphisms of topological partial groups, as arrows; $\boldsymbol{\tau}$ : The category of topological spaces, as objects and continuous maps, as arrows; $\boldsymbol{\wp}$ : A non-empty full subcategory of $\boldsymbol{\tau}$

Acknowledgments

We thank our colleagues from Al-Azhar University who provided insight and expertise that greatly assisted the research. Further, the authors are very grateful to the editor and referees for their comments and suggestions.

Funding

Not applicable.

Availability of data and materials

Not applicable

Authors' contributions

The author contributed equally to this work. The author read and approved the final manuscript. 


\section{Publisher's Note}

Springer Nature remains neutral with regard to jurisdictional claims in published maps and institutional affiliations.

Received: 15 September 2018 Accepted: 28 November 2018

Published online: 02 May 2019

\section{References}

1. Abd- Allah, A. M., Aggour, A. I., Fathy, A.: Strong semilattices of topological groups. J. Egyption Math. Soc. 24, 597-602 (2016). https://doi.org/10.1016/j.joems.2016.03.003

2. Abd-Allah, A. M., Aggour, A. I., Fathy, A.: $\underline{k}$-partial groups. J. Egyption Math. Soc. 25, 276-278 (2017). https://doi.org/ 10.1016/j.joems.2017.01.008

3. Brown, R.: Topology and Groupoids. McGraw- Hill, Oxon (2006). this updated version first published in 2006 by www. groupoids.org.uk Deganwy, United Kingdom

4. Howie, J. M.: An introduction to semigroup theory. Academic Press (1976)

5. Abd- Allah, A. M., Abdallah, M. E.-G. M.: On Clifford Semigroup,. Pur. Math. Manuscr. 7, 1-17 (1988)

6. Abd- Allah, A. M., Abdallah, M. E.-G. M.: Quotient in partial groups. Delta J. Sci. 8(2), 470-480 (1984)

7. Vogt, R. M.: Convenient category of topological spaces for algebraic topology. Proc. Adv. Study Inst. Alg. Top. Aarhus XXII, 545-555 (1970)

\section{Submit your manuscript to a SpringerOpen ${ }^{\circ}$} journal and benefit from:

- Convenient online submission

- Rigorous peer review

- Open access: articles freely available online

- High visibility within the field

Retaining the copyright to your article

Submit your next manuscript at $\gg$ springeropen.com 